\title{
Central nervous system metastases of a pulmonary epitheloid haemangioendothelioma
}

\author{
R. Díaz*, Á. Segura*, V. Calderero*, I. Cervera\#, J. Aparicio*, M.V. Jordá", L. Pellín*
}

Central nervous system metastases of a pulmonary epitheloid haemangioendothelioma. R. Díaz, Á. Segura, V. Calderero, I. Cervera, J. Aparicio, M.V. Jordá, L. Pellín. (C) ERS Journals Ltd 2004.

ABSTRACT: The case of a 55-yr-old male with a right pleural effusion and multiple bilateral nodules is reported. A diagnostic thoracothomy was necessary to obtain a definitive histological diagnosis.

During the postoperative course, the subject's neurological condition deteriorated and multiple cerebral mass lesions were discovered. The pathological analysis of both lung and cerebral tumours revealed an atypical endothelial cell proliferation; vascular immunohistochemical markers, such as factor VIII and CD34, were strongly positive. His general condition remained poor and the patient died $\mathbf{1 8}$ months after the initial diagnosis.

The final diagnosis was pulmonary epitheloid haemangioendothelioma with synchronous central nervous system dissemination, the first time the authors believe that association has been reported. Little is known of the prognosis and treatment of these tumours, due to their rarity. Negative prognostic factors appear to be the presence of symptoms, pleural effusion or multifocal presentations. Treatment should include surgical resection if possible; chemotherapy appears to have little effect. Watchful waiting is an acceptable option, especially in asymptomatic patients.

Eur Respir J 2004; 23: 483-486.
* Medical Oncology Unit, ${ }^{\#}$ Infectious Diseases Unit, and "Pathology Dept, University Hospital La Fe, Valencia, Spain.

Correspondence: Á. Segura

Medical Oncology Unit

University Hospital La Fe

Av. Campanar, 19-21

46009 Valencia

Spain

Fax: 34961973138

E-mail: segura_ang@gva.es

Keywords: Central nervous system endothelial

epitheloid haemangioendothelioma

lung

malignancy

metastases

Received: May 292003

Accepted after revision: October 62003
Epitheloid haemangioendothelioma (EHE) is a rare tumour of endothelial origin, with an intermediate course between haemangioma and conventional angiosarcoma. Its two main locations include the liver and the lung, although it has been described in many other locations. Pulmonary EHE usually manifests itself as multiple bilateral nodules in middle-aged patients and tends to follow a prolonged clinical course; dissemination outside the pulmonary parenchima is rare. The case of a 55-yr-old male with a pulmonary EHE who developed central nervous system dissemination, a unique association, is reported here. The diagnostic criteria of the malignancy and scant evidence regarding prognostic factors available in the literature are reviewed. The therapeutic options available are also presented.

\section{Case report}

A 55-yr-old male developed haemoptysis and pleuritic pain in the right hemithorax in June 2001. Apart from heavy smoking and a slight thrombocytopenia attributed to the use of metamizol, his past medical history was unremarkable. He had no history of risk factors for human immunodeficiency virus infection, tuberculosis or exposure to tuberculosis, exposure to industrial dusts or travel outside Spain. A chest radiograph performed at the University Hospital La Fe, Valencia, Spain, showed a right pleural effusion alongside multiple bilateral nodules (fig. 1). Thoracic computed tomography $(\mathrm{CT})$ confirmed these findings, revealing a $6.9 \times 4.1 \mathrm{~cm}$ liquid collection in the left lower lobe suggestive of pleural empyema and multiple bilateral nodules of different sizes, highly suggestive of metastasis (fig. 2). However, a fibrobroncoschopy failed to reveal any abnormalities, as did the pleural liquid analysis, which showed only haematic material. Two transthoracic needle biopsies of the collection were also negative for malignant cells. In order to obtain a definitive histological diagnosis, the patient was admitted to University Hospital La Fe, Valencia, Spain, in October 2001 to carry out a diagnostic thoracothomy.

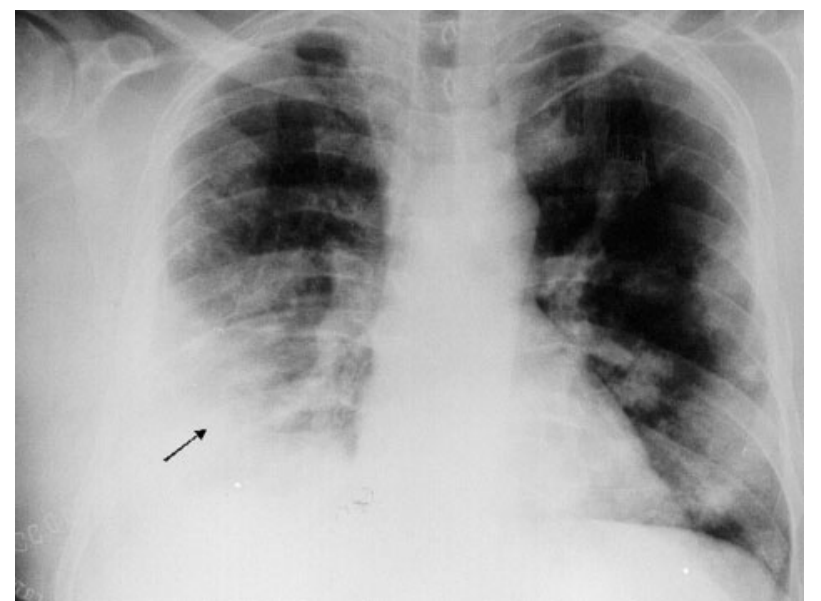

Fig. 1. - Chest radiograph shows a right sided pleural effusion (arrow) alongside multiple parenchymal nodules in both lungs. 


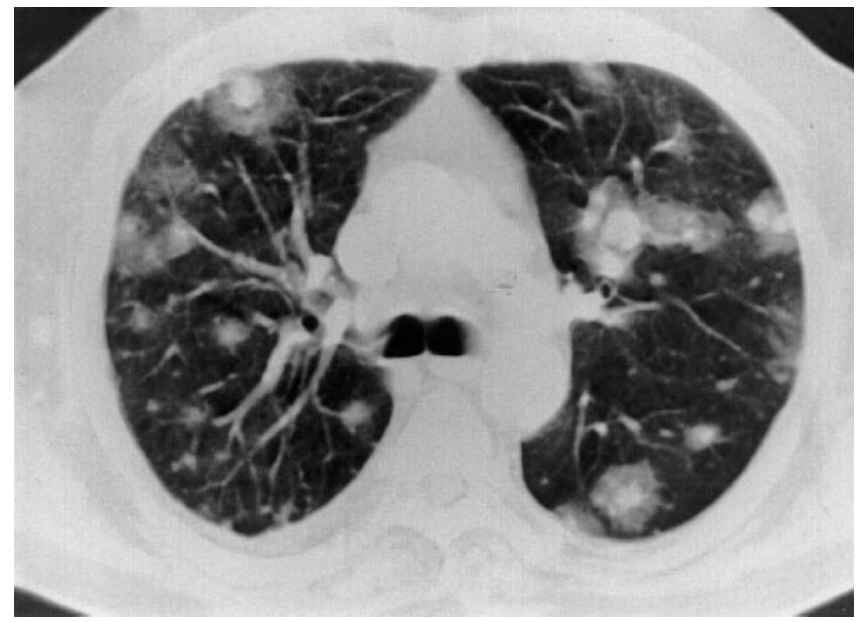

Fig. 2.-Computed tomography section shows multiple bilateral nodules of varying sizes. The nodules are well-delineated and have a homogeneous matrix.

The surgical exploration revealed a highly vascularised tumour in the left lower lobe with apparent metastasic dissemination throughout the pulmonary parenchyma. However, the initial pathological analysis of the nodules only showed foci of pulmonary infarcts with central microabscesification, surrounded with areas of organised pneumonia and endothelial and alveolar proliferation and hyperplasia; in some points, the alveolar cells showed cellular atypias and mitoses. The surrounding stroma was highly cellular, with abundant hystiocites. No evident malignant cells could be seen (fig. 3). An organised fibrinous pleuritis was also noted. All these histological changes could be secondary to systemic or infectious causes, such as mycobacteria or fungi.

The patient's clinical course soon deteriorated with the appearance of fever and headache. The neurological examination showed nominal aphasia, right homonimous hemianopsya and a severe gait impairment. A brain magnetic resonance imaging showed multiple subcortical hemispheric lesions with ring morphology and surrounding oedema (figs 4 and 5). Analysis of the cerebrospinal fluid showed no initial abnormalities. With the suspicion of disseminated mycobacterial infection, empirical treatment with dexamethasone, isoniazid, rifampicin and etambuthol was begun. Due to

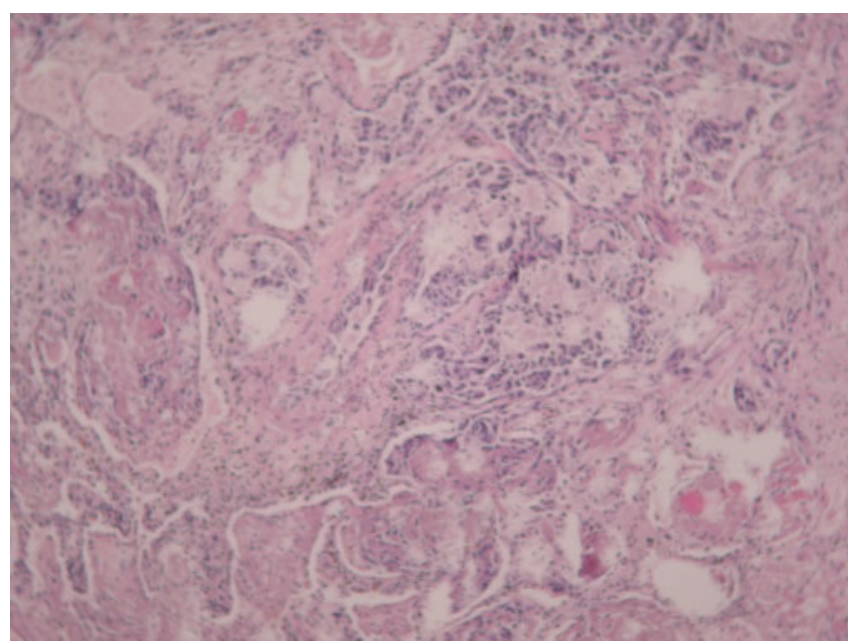

Fig. 3.-The histological analysis of the lung tumour, with the haematoxilin-eosin stain, shows several areas of coagulative necrosis surrounded by areas of intense alveolar and endothelial proliferation.

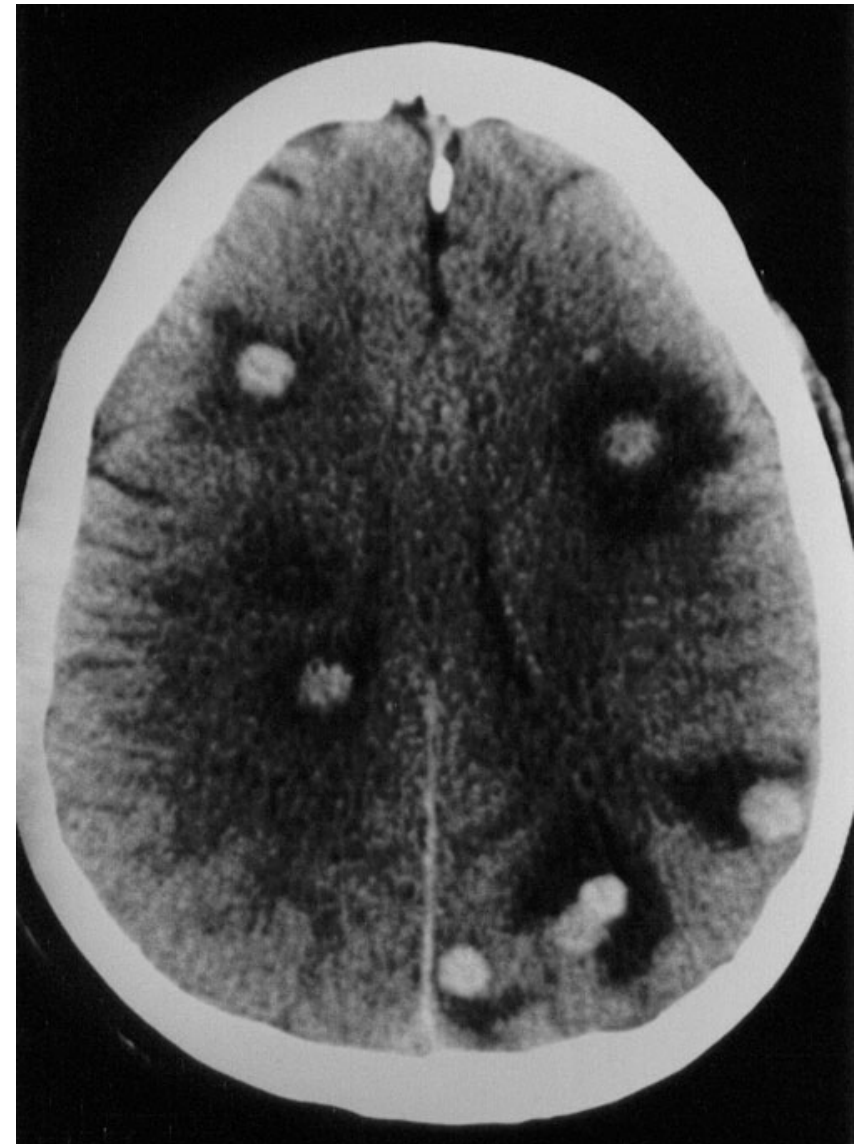

Fig. 4.-Computed tomography section of the brain shows multiple nodular lesions strongly suggestive of metastasis.

the extremely unusual evolution, a biopsy of one of the brain lesions was performed.

The brain biopsy showed the same endothelial proliferation as the lung lesion with atypical cells and some mitoses. The immunohistochemical studies performed in both biopsies (including the alveolar component of the lung lesion) showed negativity for cytokeratin, epithelial membrane antigen (EMA) and thyroid transcription factor 1 (TTF-1) and strong positivity for vimentin and the vascular markers factor VIII and CD34 (fig. 6). All microbiological studies for mycobacteria, fungi and bacterial pathogens were negative. A standard battery of autoantibodies was also negative. Thus, the final pathological diagnosis was pulmonary EHE with central nervous system dissemination.

The clinical state of the patient remained poor, with an Eastern Cooperative Oncology Group performance status of 3. Symptomatic treatment with corticosteroids and major opioids was begun. Holocraneal irradiation for a total dose of 30 Grays was given, with little improvement in his neurological impairment. He remained wheelchair-bound until his death in January 2003, 18 months after the initial diagnosis.

\section{Discussion}

EHE is a rare tumour of endothelial origin, which has been described primarily in the liver [1] and in the lung [2]. In its pulmonary form, it was originally described by DAIL and LIEBOW [3] as "intravascular bronchioalveolar tumour"; these authors noted the tumour's remarkable propensity to invade pulmonary vessels and small airways. Since then, $<50$ cases 


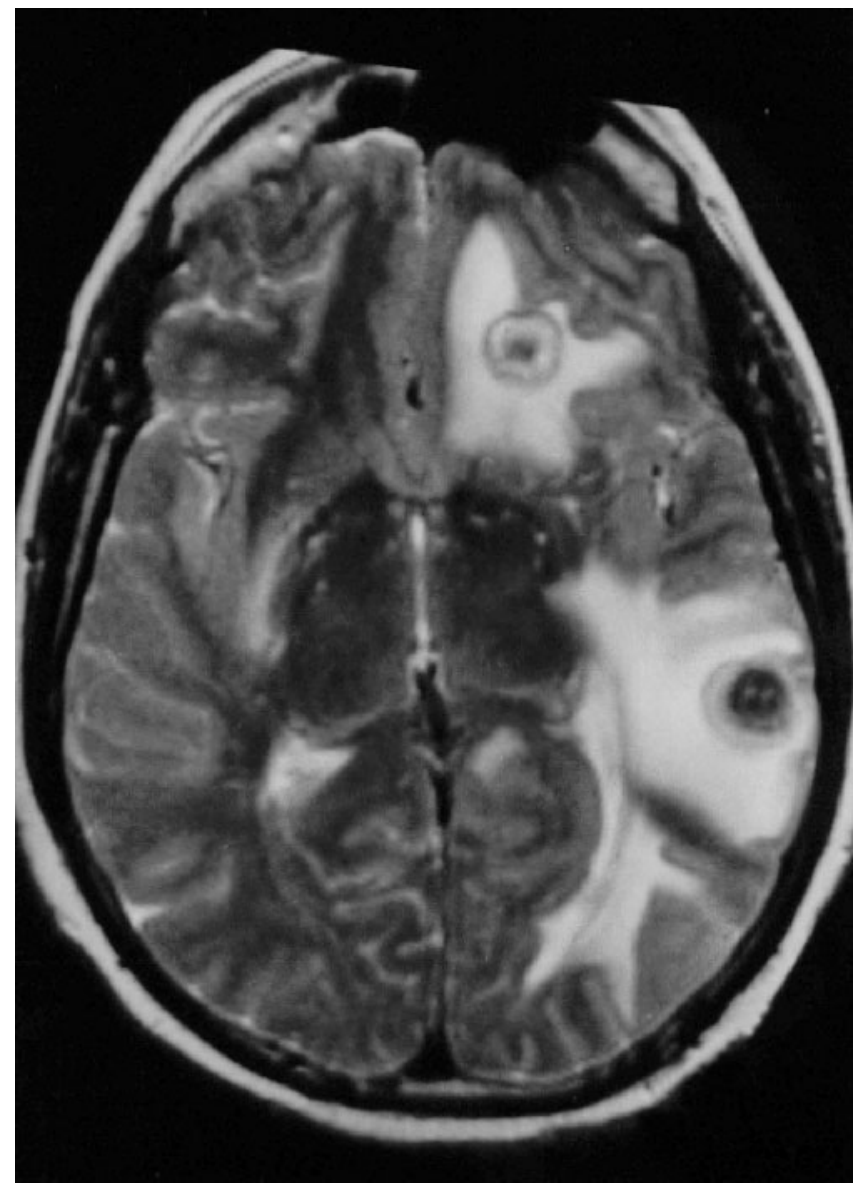

Fig. 5.-T1-weighted magnetic resonance imaging section through the brain shows multiple nodular lesions with ring-like morphology. Note that all lesions are surrounded by perifocal oedema.

have been described in the literature worldwide. It is a tumour of borderline malignancy, which tends to follow an intermediate course between haemangioma and conventional angiosarcoma [4].

In the typical pulmonary forms, EHE presents with nonspecific symptoms, such as chest pain, cough or sputum, or it

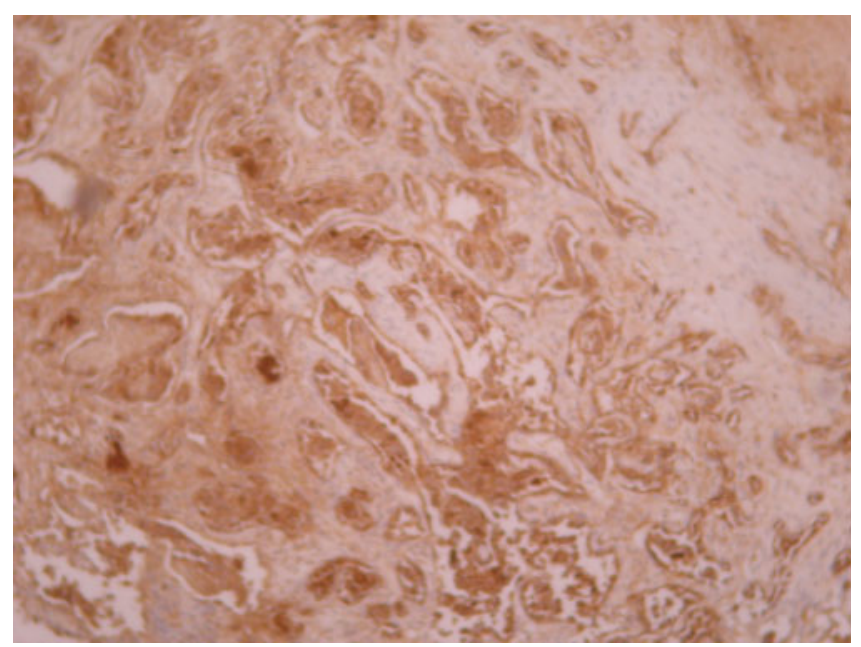

Fig. 6. - The immunohistochemical study for factor VIII presence in the lung tumour shows intense positivity for this vascular marker. may remain asymptomatic [2, 5]. It usually affects middleaged patients, with a female predominance, although cases in children and in the elderly have been described $[2,5,6]$. Multifocal nodular densities up to $2 \mathrm{~cm}$ in diameter, with well- or ill-defined margins, are the most common feature of pulmonary EHE on chest radiography [2, 7]; these are often mistaken for metastatic disease but they show little or no growth on serial radiography. The presence of a pleural effusion or hiliar or mediastinal lymphadenopathy is rare [7, 8]. The thoracic CT usually reveal more nodules that those in the chest radiography. In long-standing cases, extensive calcification of the nodules and insterstitial involvement can be seen [9].

The pathological characteristics of pulmonary EHE are more distinctive in the lung than in other locations because of the zonal pattern of the pulmonary nodules. The centre of the nodule is typically sclerotic, necrotic and hypocellular and it may resemble an infarct, a microabscess, a hyalinised granuloma or even a hamartoma. The periphery of the nodule is composed of an hypercellular and sclerotic stroma and clusters of neoplastic cells with few morphological signs of malignancy that grow as polypoid tufts into alveolar spaces $[2,4,5,10,11]$. Many autoimmune and infectious processes can produce a similar pathological picture of central coagulative necrosis and atypical endothelial and intra-alveolar cell growth, so all these causes should be ruled out before a definitive diagnosis is made [12].

In this case, the immunohistochemical study was pivotal for the correct diagnosis, showing negativity for epithelial markers, such as EMA, TTF-1 and cytokeratin, and strong positivity for vimentin (a mesenchymal marker), factor VIII and CD34 (both vascular markers). All microbiological studies were negative, thus ruling out infection by mycobacteria or fungi.

Due to the rarity of the tumour, its natural history is difficult to predict. In typical pulmonary cases, it appears to follow a protracted clinical course, with death after a variable length of time usually resulting from respiratory insufficiency due to substitution of the healthy pulmonary parenchima $[2,4,5,13]$. However, some authors have observed partial spontaneous regressions of the lesions [5]. Lymphatic dissemination is extremely rare, supporting the endothelial origin of the tumour. Haematogeneous metastases are also rare and have been described especially in the liver but also in the skin, serosa, spleen, tonsils, retroperitoneum and kidney $[2,5,10,14,15]$. However, the authors believe this is the first case reported of a pulmonary EHE with a synchronous massive dissemination to the central nervous system.

Prognostic factors in pulmonary EHE have been difficult to validate, due to the small number of patients involved. In DAIL et al. [2], a report of 17 patients with pulmonary EHE, negative prognostic factors appeared to be respiratory symptoms at presentation, extensive lymphangitic spread, pleural invasion, hepatic metastases and peripheral lymphadenopathy. In the largest series (21 patients) published to date by KITAICHI et al. [5], an unfavourable prognosis was associated with pleural effusion on chest radiography, fibrous pleuritis with extrapleural proliferation of tumour cells and spindle tumour cells at histology; surprisingly, liver involvement was not associated with a worse prognosis.

Treatment can vary from observation in asymptomatic patients, surgery in the few patients with resectable disease or chemotherapy in widely disseminated disease $[2,4,5,10,13]$; these options should be discussed with the patient. However, the chemotherapy to be used is still a matter of debate. The notion of pulmonary EHE as a chemoresistant disease is probably still true $[2,5]$. The authors have only found a case of complete remission of a pleural EHE after treatment with chemotherapy (carboplatin and etoposide) [16] and a partial 
remission of a widely disseminated EHE with a year's treatment of $\alpha-2 \mathrm{~A}$ interferon, suggesting a possible role of immunotherapy [17].

In conclusion, this case study reports the first case of a pulmonary epitheloid haemangioendothelioma with synchronous central nervous system dissemination. Prognosis of this disease, especially if disseminated, remains poor and treatment is usually unsatisfactory. Novel therapeutic strategies are urgently needed, which could include new chemotherapeutic agents or the use of immunotherapy.

\section{References}

1. Lauffer JM, Zimmermann A, Krahenbuhl L, Triller J, Baer $\mathrm{Hu}$. Epitheloid hemangioendothelioma of the liver. Cancer 1996; 78: 2318-2327.

2. Dail DH, Liebow AA, Gmelich JT, et al. Intravascular, bronchiolar, and alveolar tumor of the lung (IVBAT): an analysis of twenty cases of a peculiar sclerosing endothelial tumor. Cancer 1983; 51: 452-462.

3. Dail DH, Liebow AA. Intravascular bronchioalveolar tumor. Am J Pathol 1975; 78: 6-7.

4. Bollinger BK, Laskin WB, Knight CB. Epitheloid hemangioendothelioma with multiple site involvement. Cancer 1994; 73: 610-615.

5. Kitaichi $\mathrm{M}$, Nagai S, Nishimura $\mathrm{K}$, et al. Pulmonary epitheloid haemangoendothelioma in 21 patients, including three with partial spontaneous regression. Eur Respir J 1998; 12: 89-96.

6. Rock MJ, Kaufman RA, Lobe TE, Hensley SD, Moss ML. Epitheloid hemangioendothelioma of the lung (intravascular bronchioloalveolar tumor) in a young girl. Pediatr Pulmonol 1991; 11: 181-186.

7. Mata JM, Cáceres J, Prat J, López JI, Velilla O. Intravascular bronchio-alveolar tumor: radiographic findings. Eur J Radiol 1991; 12: 95-97.

8. Mukundan G, Urban BA, Askin FB, Fishman EK Pulmonary epitheloid hemangioendothelioma: atypical radiologic findings of a rare tumor with pathologic correlation. J Comput Assist Tomogr 2000; 24: 719-720.

9. Luburich P, Ayuso MC, Picado C, Serra-Batllés J, Rámirez JF, Solé M. CT of pulmonary epitheloid hemangioendothelioma. J Comput Assist Tomogr 1994; 20: 562-565.

10. Miettinen M, Collan Y, Halttunen P, Maamies T, Vilkko P. Intravascular bronchioloalveolar tumor. Cancer 1987; 60: 2471-2475

11. Weiss SW, Ishak KG, Dail DH, Sweet DE, Enzinger FM. Epitheloid hemangioendothelioma and related lesions. Sem Diag Pathol 1986; 3: 259-287.

12. Case Records of the Massachusetts General Hospital. Weekly clinicopatological exercises. Case 6-2000. Hemoptysis in a 20 -year-old man with multiple pulmonary nodules. $N$ Engl J Med 2000; 342: 572-578.

13. Ledson MJ, Convery R, Carty A, Evans CC. Epitheloid hemangioendothelioma. Thorax 1999; 54: 560-561.

14. Yanagawa $\mathrm{H}$, Hashimoto $\mathrm{Y}$, Bando $\mathrm{H}$, Takishita $\mathrm{Y}$, Nagano T. Intravascular bronchioloalveolar tumor with skin metastases. Chest 1994; 105: 1882-1884.

15. Siga K, Tateda M, Saijo S, Yokoyama J. A case report of epitheloid hemangioendothelioma metastasizing to the tonsil. Nippon Jibiinkoka Gakkai Kaiho 2002; 105: 937940

16. Pinet C, Magnan A, Garbe L, Payan MJ, Vervloet D. Aggressive form of pleural epitheloid hemangioendothelioma: complete response after chemotherapy. Eur Respir $J$ 1999; 14: 237-238.

17. Roudier-Pujol C, Enjolras O, Lacronique J. Multicentric epitheloid hemangioendothelioma in partial remission with interferon treatment. Ann Dermatol Venereol 1994; 121: 898 904. 